\title{
Novel Possibility of Nonstandard Statistics in the Inflationary Spectrum of Primordial Inhomogeneities
}

\author{
Gabriel LEÓN ${ }^{\dagger}$ and Daniel SUDARSKY ${ }^{\ddagger}$ \\ $\dagger$ Department of Physics, University of Trieste, Strada Costiera 11, 34014 Trieste, Italy \\ E-mail: gabriel.leon@nucleares.unam.mx \\ ¥Instituto de Ciencias Nucleares, Universidad Nacional Autónoma de México, \\ México D.F. 04510, México \\ E-mail: sudarsky@nucleares.unam.mx
}

Received August 31, 2011, in final form April 16, 2012; Published online April 21, 2012

http://dx.doi.org/10.3842/SIGMA.2012.024

\begin{abstract}
Inflation is considered one of the cornerstones of modern cosmology. However, the account of the origin of cosmic structure, as provided by the standard inflationary paradigm, is not fully satisfactory. The fundamental issue is the inability of the usual account to point out the physical mechanism responsible for generating the inhomogeneity and anisotropy of our Universe, starting from the exactly homogeneous and isotropic vacuum state associated with the early inflationary regime. We briefly review this issue here together with the proposal to address this shortcoming in terms of a dynamical collapse of the vacuum state of the inflaton field, which has been considered in previous works. The main goal of this manuscript is to discuss certain statistical aspects associated with the collapse and its implications in the primordial spectrum, particularly those connected with the possible appearance of a novel type of unusual correlations.
\end{abstract}

Key words: inflation; cosmology; quantum gravity

2010 Mathematics Subject Classification: 83F05; 81T20; 81P05

\section{Introduction}

The inflationary paradigm represents one of the pillars of the $\Lambda$ CDM cosmological model. It was initially proposed as a solution to the classical naturalness problems of the the big bang models $[1,13,15,18,28,40]$, but its impact became even more significant when it came to be regarded as the natural mechanism to account for the seeds of cosmic structure [14, 16, 17, 31, 32, 41]. However, as originally presented in [37], the standard picture suffers from a conceptual drawback: we know that our Universe is quantum mechanical and, thus, the classical descriptions must be regarded as nothing but short-hand and imprecise characterizations of complicated quantum mechanical states. The Universe that we inhabit today is certainly very well described at the classical level by an inhomogeneous and anisotropic classical state. That is, such description must be nothing but a concise and imperfect description of an equally inhomogeneous and anisotropic quantum state. Consequently, if we want to seriously consider a theory, in which the early quantum state of the Universe was perfectly symmetric (the symmetry being the homogeneity and isotropy), then we need to provide and explanation for why the quantum state that describes our actual Universe lacks such symmetries. Since there is nothing in the dynamical evolution (as given by the standard inflationary approach) of the quantum state that can break those

\footnotetext{
*This paper is a contribution to the Special Issue "Loop Quantum Gravity and Cosmology". The full collection is available at http://www.emis.de/journals/SIGMA/LQGC.html
} 
symmetries, then we are left with an incomplete theory. Therefore a natural inquiry arises: How does the actual inhomogeneities in the Universe we inhabit emerged?. The standard and phenomenologically successful accounts on this matter rely on the identification of the quantum fluctuations of certain observables associated to a homogeneous and isotropic Universe, with the averages over an ensemble of inhomogeneous Universes of their analogue classical quantities, i.e. the identification of quantum uncertainties and classical stochastic perturbations. Nevertheless, as discussed in [37, 44], one can not avoid concluding that, using standard physics, there is no clear justification for such kind of identification. In fact, these shortcomings have been recognized by others in the literature $[29,30,33,47]$.

The idea that has been considered in previous works [37, 42, 43, 44, 45], as a possibility to deal with that problem, involves adding to the standard inflationary model the hypothesis that the collapse of the wave function is an actual physical process that occurs independently of external observers. The collapse hypothesis relies on the idea that something that effectively might be described in terms of such a collapse of the wave function could have its origins in the passage from the atemporal regime of quantum gravity to the classical space-time description underlining the general theory of relativity. That is, in going from one description to the other, we might be forced to characterize some aspects of the underlying physics in terms of sudden jumps which are not compatible with the unitary Schrödinger evolution, and which modify the state of the Universe in an stochastic way. Therefore, being capable of transforming a condition that was initially homogeneous and isotropic into another one that is not. It is worth mentioning that, in fact, conceptually similar ideas have been considered by many physicists concerned with the measurement problem in quantum mechanics [3, 12, 34], and the proposal that some quantum aspects associated to the gravitational interaction might lie at the root of this effect only serves to make the idea even more attractive [8, 9, 35, 36].

The detailed discussion of the conceptual problems and those associated with the standard inflationary paradigm have been presented in previous works by some of us and by others in $[25,34,37,44]$. We will not reproduce those arguments here. On the other hand, a detailed discussion of the statistical aspects concerning both our modified proposal involving the quantum collapse of the wave function and as well as various aspects of the standard picture are presented in [25]; and again we will not reproduce most of those arguments here. In this paper our aim is to present one novel possibility regarding the statistical aspects which might be present in the primordial spectrum. It would correspond to the existence of non-trivial correlations between the random variables characterizing the collapse of certain modes and its statistical properties. In particular, these features would give raise to a very particular deviation from the flat shape of the spectrum of the primordial fluctuations, and thus to a characteristic signature temperature anisotropies in the CMB. This is a crucial difference with the standard accounts, as the distribution of the different quantum field modes has previously been assumed to be Gaussian, i.e. that all the fundamental variables are uncorrelated.

As we will show next, the consideration of this possibility is enabled by the fact that the statistical aspects, in the collapse picture, are quite transparent and clearly identifiable. In fact, the motivation for the work presented in this article, is the formal and rigorous analysis presented in [7]. There, the collapse hypothesis is formally considered within the full framework of Quantum Field Theory in curved space-times, as this is the most reliable approach we can take in the absence of a complete theory of quantum gravity.

The paper is organized as follows. In Section 2, we will review some of the considerations contrasting the collapse proposal and the standard approach. We give a brief account of way we think that these ideas would fit into the more standard views of the quantum/gravity interface. We also introduce the Semiclassical Self-consistent Configuration program which is the main motivation behind the results presented in this article. In Section 3, we review the collapse models description for the inflationary origin of the seeds of the cosmic structure. In Section 4, 
we present how the stochastic nature of the collapse proposal might induce new statistical features in the observable spectrum of anisotropies in the CMB. Finally, in Section 5, we discuss our findings. The conventions we will be using include a $(-,+,+,+)$ signature for the spacetime metric. We will use units where $c=1$, but will keep the gravitational constant $G$ and $\hbar$ explicit throughout the paper.

\section{General framework of the collapse model}

The collapse model for the emergence of the seeds of structure during inflation was originally proposed in [37], and since then has been further developed [5, 6, 24, 26]. In this section we will present a review of the ideas that we have explored and which are connected to the signature of novel statistical features explored in this paper.

Within the standard approach [2, 20,21, 27, 48], the possibility of deviations from simple statistics, is often referred as the study of non-Gaussianity, and is a feature thought to be characterized only in the higher order statistical $n$-point functions. We will discuss a novel type of deviation from the standard statistics that is suggested by our approach and that could lead to modifications directly observable in CMB the spectrum.

In the standard inflationary paradigm, the perturbations of the field $\delta \phi$ and the perturbations of the curvature $\Psi$ are treated as standard quantum fields ${ }^{1}$ evolving in a classical quasi-De Sitter background space-time. The statistical aspects involve a series of identification between quantum $n$-point functions, statistical $n$-point functions for ensembles of universes and averages over our universe. Those issues are often left unspecified and the clear characterization of these aspects in the theoretical analysis is often missing. On the other hand, within the collapse proposal, it becomes quite evident where the stochastic nature of the problem resides. That is, the dynamical collapse of the wave function is taken to be a physical mechanism governed essentially by the stochastic rules inherent to the Quantum Theory. Although, we of course do not know what is the physics behind the collapse, we can parameterize it (in the next section we will detail this parametrization) and hope to learn something about its basic characteristics. The important point is that, if the initial state (say the Bunch-Davies (BD) vacuum state) collapses (or is reduced) to a different state, which lacks the symmetries of the original one, and in a manner in which nontrivial correlations might be generated, then the energy momentum tensor of this resulting state would lead, not only to the emergence of anisotropies and inhomogeneities, but might result in a particular type of signature appearing in the primordial spectrum of fluctuations as observable features. We will explain in the next sections how such primordial non-flat spectrum could arise, with the departures from flatness representing the novel signatures we have been discussing.

\subsection{The semiclassical self-consistent configuration}

As mentioned in the Introduction, the inflationary scenario is a very peculiar setting where one can explore some of the fundamental aspects of modern physics. In particular, it provides us with a situation in which the effects of gravity and quantum mechanics are strongly coupled. However, we do not have a complete theory of Quantum Gravity (QG). In fact, one of the problems that arises when trying to unify General Relativity with Quantum Mechanics is the so called problem of time in quantum gravity [19].

It is a known fact that the proposals for the canonical Quantization of Gravity leads to timeless theories. This is simply because the lapse and shift vectors, naturally associated with the notion of time in General Relativity, disappear from the theory (the technical reason is that

\footnotetext{
${ }^{1}$ In fact they are both treated as part of a unified field $v$.
} 
the physical states of the theory need to satisfy the Hamiltonian and momentum constraints). The Loop Quantum Gravity approach (LQG), as the modern version of such ideas, does not escape from such problem. Let us analyze in brief detail our view on how to deal with it and its relation with our proposal (see [7, 43]).

In LQG, one starts with the characterization of gravity in terms of the canonical variables given by he triad $E_{i}^{a}$ and the connection variables $A_{a}^{i}$. These variables are serve to describe the geometry of a 3-spatial hypersurface, and characterize its embedding in a 4-dimensional (spacetime) manifold. At the quantum level, however, one passes to a description where the basic operators are associated with holonomies and fluxes $\left(\mathcal{U}_{\gamma}, \mathcal{F}_{s}\right)$ which correspond to integrals of the connection and triads over closed paths $\gamma$ and 2-surfaces $s$ respectively. The quantum states of the theory are thus characterized in terms of these variables, and the recovery of space-time notions from this setting is rather non trivial. In fact it seems likely one would need special kinds of semiclassical states which nevertheless should be solutions of the constraints of the theory including the Hamiltonian constraint. It is still a challenge to construct these states and the full recovery of space-time from them seems to be further away. However we can try to envision how this process would look when such technical difficulties are overcome.

Consider now, that we have incorporated into the theory, along with the geometrical variables, some matter fields, each described by a pairs of cannonical variables $\left(\varphi_{i}, \pi_{i}\right)$. In most attempts to recover time, the crucial step is the construction a quantum variable $\hat{T}\left(\varphi_{i}, \pi_{i}, \mathcal{U}_{\gamma}, \mathcal{F}_{s}\right)$, which might depend on both the matter and gravitational degrees of freedom, and which shouls play the role of as a "physical clock". On the other hand, we assume we are given a wave function for the configuration variables $\Phi\left(\varphi_{1}, \ldots, \varphi_{n}, U_{\gamma}\right)$. Following the canonical quantization procedure, the wave function $\Phi$ must satisfy the Hamiltonian constrain, i.e. $\hat{H}_{\alpha} \Phi\left(\varphi_{1}, \ldots, \varphi_{n}, U_{\gamma}\right)=0$; $\alpha=0,1,2,3, \ldots$. One can then obtain an effective wave function $\Psi$ for the remaining variables by using a projection operator $\hat{P}_{T,[t, t+\delta t]}$. The projector operator acts on the wave function $\Phi$ projecting it onto the subspace where the spectrum of $\hat{T}\left(\varphi_{i}, \pi_{i}, \mathcal{U}_{\gamma}, \mathcal{F}_{s}\right)$, corresponding to the region between $t$ and $t+\delta t$, acquires some particular values. After an effective wave function $\Psi(t)$ has been obtained by this procedure, one should be able, in principle, compute the expectations values of geometrical operators like areas and volumes (as well as others) to characterize the spatial metric and extrinsic curvature associated with the "hypersurface" corresponding to the selected value of $t$. We would do this for a large enough range of such values and obtain data characterizing the space-time in terms of data for a slicing. In other words, the procedure described allows us to obtain an "average" $3+1$ decomposition for the space-time, where the slicing would be identified with the hypersurfaces on which the geometrical quantities are given by the expectation values (calculated using the wave function $\Psi(t)$ ). Therefore, one would be able to use the standard lapse and shift functions to specify the space-time and the slicing. The aspect of this approach that we want to focus on, is that, as shown in [10,11], the evolution of $\Psi(t)$ would be given by a modified version of Schrödinger's equation including departures from the standard unitary evolution. Thus it is not unnatural that an effective sort of Schrödinger's equation, involving something that looks, from the effective point of view, as a collapse of the wave-function, could find its fundamental explanation in the setting we have proposed. In fact, these types of modifications have been previously proposed, with different motivations, and in different contexts, by the Quantum Foundations community leading to the so called collapse models [3, 12, 34, 35, 36].

As we do not have at this stage a complete workable theory of QG, the framework under which our proposal has been studied is directly that of semiclassical general relativity, which we have taken to be just an effective description of limited range of applicability, and which might well be the only setting where standard space-time notions can be recovered from an underlying quantum gravity theory. However, we will assume that its applicability includes the inflationary cosmological regime which is the situation of interest to us here. As we have shown in [7], the 
nature of the formal description one needs to consider, even though we do not take it to be in any sense fundamental, is not as trivial as one might initially imagine.

In that approach, one considers that the Universe can be described, by what we call a Semiclassical Self-consistent Configuration (SSC). That is, a space-time geometry characterized by a classical space-time metric and a standard quantum field theory constructed on that fixed space-time background, together with a particular state in that construction such that the semiclassical Einstein equations hold. In other words, we will say that the set $\left\{g_{\mu \nu}(x), \hat{\varphi}(x), \hat{\pi}(x)\right.$, $\mathscr{H}|\xi\rangle \in \mathscr{H}\}$ represents a SSC if and only if $\hat{\varphi}(x), \hat{\pi}(x)$ and $\mathscr{H}$ correspond to a quantum field theory constructed over a space-time with metric $g_{\mu \nu}(x)$ (as described in, say [46]), and the state $|\xi\rangle$ in $\mathscr{H}$ is such that

$$
G_{\mu \nu}[g(x)]=8 \pi G\left\langle\xi\left|\hat{T}_{\mu \nu}[g(x), \hat{\varphi}(x), \hat{\pi}(x)]\right| \xi\right\rangle,
$$

for all the points in the space-time manifold.

Such description is thought to be appropriate in the regime of interests except in those times when a collapse occurs. The resulting description is such that when the initial situation is homogeneous and isotropic, the evolution can not generate any departure from that symmetry. The emergence of inhomogeneities and anisotropies requires therefore something akin to a collapse process, something which in turn clearly necessitates a modified description. In [7] we developed what we consider to be a natural prescription for the description of such collapses, and we analyzed in detail the emergence through such process of an excitation of a single mode.

The proposal is as follows: One considers, first within the Hilbert space associated to the given SSC-i, that a transition $\left|\xi^{(\mathrm{i})}\right\rangle \rightarrow\left|\zeta^{(\mathrm{i})}\right\rangle_{\text {target }}$ "is about to happen", with both $\left|\xi^{(\mathrm{i})}\right\rangle$ and $\left|\zeta^{(\mathrm{i})}\right\rangle_{\text {target }}$ in $\mathscr{H}^{(\mathrm{i})}$. Generically, the set $\left\{g^{(\mathrm{i})}, \hat{\varphi}^{(\mathrm{i})}, \hat{\pi}^{(\mathrm{i})}, \mathscr{H}^{(\mathrm{i})}\left|\zeta^{(\mathrm{i})}\right\rangle_{\text {target }}\right\}$ will not represent a new SSC. In order to have a sensible picture, our recipe is, as discussed in [7], to relate this state $\left|\zeta^{(\mathrm{i})}\right\rangle_{\text {target }}$ with another one $\left|\zeta^{(i i)}\right\rangle$ "living" in a new Hilbert space $\mathscr{H}^{(\mathrm{ii})}$ for which $\left\{g^{(\mathrm{ii})}, \hat{\varphi}^{(\mathrm{ii})}, \hat{\pi}^{(\mathrm{ii})}, \mathscr{H}^{(\mathrm{ii})}\left|\zeta^{(\mathrm{ii})}\right\rangle\right\}$ is an actual SSC. We denote the new SSC by SSC-ii. Thus, first we need to determine the "target" (non-physical) state in $\mathscr{H}^{(\mathrm{i})}$ to which the initial state is in a sense "tempted" to jump, and after that, one relates such target state with a corresponding state in the Hilbert space of a new SSC, the SSC-ii. Following our previous treatments on the subject (see for instance [37]), one then considers that the target state is chosen stochastically, guided by the quantum uncertainties of designated field operators, evaluated on the initial state $\left|\xi^{(i)}\right\rangle$, at the collapsing time.

It is natural to assume that after the collapse, the expectation values of the field and momentum operators in each mode will be related to the uncertainties of the pre-collapse state (recall that the expectation values in the vacuum state are zero). In the vacuum state, the operators $\hat{y}_{\vec{k}}$ and $\hat{\pi}_{\vec{k}}$ are individually characterized by Gaussian wave functions centered at 0 with widths (uncertainties) $\left(\Delta \hat{y}_{\vec{k}}\right)_{0}^{2}$ and $\left(\Delta \hat{\pi}_{\vec{k}}\right)_{0}^{2}$, respectively. The collapse proposals that we have been considering are based on the hypothesis that each mode would jump to a new state where the expectation value would be determined by both, the scale of the uncertainties and some random variable. The idea is that the collapse would be a process taking place at a certain time, and resembling an imprecise measurement, which, of course, would be brought upon by novel physics requiring no observer or measuring apparatus.

A more precise characterization of the collapse process maintains the essence of such prescription, but now takes into account that the change of the state would need to be accompanied by a change in the space-time metric and that would require a new Hilbert space, and a new quantum state in that Hilbert space. As shown in [7], the scheme serves to characterize a complete post-collapse SSC (i.e. the SSC-ii). In fact, regarding the identification between the two different SSC's involved in the collapse, we took in [7] what turned out to be a very promising prescription: Consider that the collapse takes place along a Cauchy hypersurface $\Sigma$. A transition from the physical state $\left|\xi^{(\mathrm{i})}\right\rangle$ in $\mathscr{H}^{(\mathrm{i})}$ to the physical state $\left|\zeta^{(\mathrm{ii})}\right\rangle$ in $\mathscr{H}^{(\mathrm{ii})}$ (associated to a certain 
target non-physical state $\left|\zeta^{(\mathrm{i})}\right\rangle_{\text {target }}$ in $\left.\mathscr{H}^{(\mathrm{i})}\right)$ will occur in a way that

$$
\left.\operatorname{target}\left\langle\zeta^{(\mathrm{i})}\left|\hat{T}_{\mu \nu}^{(\mathrm{i})}\left[g^{(\mathrm{i})}, \hat{\varphi}^{(\mathrm{i})}, \hat{\pi}^{(\mathrm{i})}\right]\right| \zeta^{(\mathrm{i})}\right\rangle_{\text {target }}\right|_{\Sigma}=\left.\left\langle\zeta^{(\mathrm{ii})}\left|\hat{T}_{\mu \nu}^{(\mathrm{ii})}\left[g^{(\mathrm{ii})}, \hat{\varphi}^{(\mathrm{ii})}, \hat{\pi}^{(\mathrm{ii})}\right]\right| \zeta^{(\mathrm{ii})}\right\rangle\right|_{\Sigma},
$$

i.e. in such a way that the expectation value of the energy momentum tensor, associated to the states $\left|\zeta^{(\mathrm{i})}\right\rangle_{\text {target }}$ and $\left|\zeta^{(\mathrm{ii})}\right\rangle$ evaluated on the Cauchy hypersurface $\Sigma$, coincides. Note that the left hand side in the expression above is meant to be constructed from the elements of the SSC-i (although $\left|\zeta^{(\mathrm{i})}\right\rangle_{\text {target }}$ is not really the state of the SSC-i), while the right hand side correspond to quantities evaluated using the SSC-ii.

In the situation of interest studied in [7], the SSC-i corresponded to the homogenous and isotropic space-time characterized by $\Psi=0$ with the state of the quantum field corresponding to the BD vacuum. Meanwhile, the SSC-ii corresponded to an excitation of a single mode (with comoving wave number $\left.\vec{k}_{0}\right)$, characterized by a Newtonian potential given by $\Psi=F(\eta) \cos \left(\vec{k}_{0} \cdot \vec{x}\right)$, and a specific quantum state for the inflaton field. The energy momentum tensor for that state is compatible with this space-time metric according to the SSC recipe.

The point is that, as shown in detail in [7], when the SSC-ii corresponds to a potential including spatial dependences with wavenumber $\vec{k}_{0}$ the normal modes of the corresponding Hilbert space $\mathscr{H}^{(\mathrm{ii})}$, which would otherwise be characterized by the typical spatial dependence $e^{i \vec{k} \cdot \vec{x}}$, and would be called the $\vec{k}$ modes, would now contain also corrective contributions of the form $e^{i\left(\vec{k} \pm \vec{k}_{0}\right) \cdot \vec{x}}$.

In turn, this implies that if we want to ensure that the energy momentum tensor does not lead to the excitation of other modes besides the $\pm \vec{k}_{0}$ in the Newtonian potential, then the state of the quantum field needs to be excited, not only in the modes $\pm \vec{k}_{0}$, but also in the modes $\pm n \vec{k}_{0}$, with $n$ integer (with the degree of excitation decreasing with increasing $n$ ). In those circumstances, one can not longer argue that at the time when these higher modes collapse, there should be complete symmetry in the a priori statistical distribution of the post-collapse parameters of the state.

That is, there seems to be ample justification to assume that the value of the random parameter $x_{n \vec{k}_{0}}(n \neq 1)$ would not be completely independent from the value taken by the random parameters $x_{\vec{k}_{0}, y}$ and $x_{\vec{k}_{0}, \pi}$. Focussing for simplicity on the modes, which in view of the preceding discussion one expects to be more closely correlated, we limit hereafter consideration to the case $n=2$, and characterize the level of correlation by an unknown quantity we label $\varepsilon$. We will show in detail how these ideas are implemented in Section 4.

We should note here we will not be using at this point the full fledged formal treatment developed in [7]. This is because, as can be seen there, the problem becomes extremely cumbersome even in the treatment of a single mode. Thus, even though it is in principle possible to use such detailed formalism to treat the complete set of relevant modes, when studying the CMB spectrum the task quickly becomes a practical impossibility. We will instead rely on the less formal treatments we had employed in previous works, and retain from the results of [7] only the unusual correlations we discussed above. Before doing so, we will briefly review the basic details, of the simple version of our formalism. The reader should be warned that this is not (and can not expected to be) a fully self contained work, as doing that would involve repeating extensive discussions and analysis already published in the literature.

\section{The collapse model for the inflationary field}

The starting point of the specific analysis is the same as the standard picture, i.e. the action of a scalar field coupled to gravity:

$$
S=\int d^{4} x \sqrt{-g}\left[\frac{1}{16 \pi G} R[g]-1 / 2 \nabla_{a} \phi \nabla_{b} \phi g^{a b}-V(\phi)\right],
$$


where $\phi$ stands for the inflaton and $V$ for the inflaton's potential. One then splits both, metric and scalar field into a spatially homogeneous (background) part and an in-homogeneous part (fluctuation), i.e. $g=g_{0}+\delta g, \phi=\phi_{0}+\delta \phi$.

Note that in the full fledged formalism introduced in [7], the quantum description is applied, not only to the inflaton mode perturbation, but also to the "background field" which is characterized in terms of the zero mode of a quantum field theory construction on the inflating space-time. At the same time, the metric sector is treated exclusively at the classical level as corresponding to the semiclassical description we have relied on (for more on the justification for this see [7]). Here however, we will return to the simpler but more convenient formalism of previous treatments, retaining as we have said only the interesting novel correlations we discussed. Thus, here the zero mode of the inflaton field will be treated as a classical background field.

The background is taken to be the spatially flat Friedmann-Robertson Universe with line element $d s^{2}=a(\eta)^{2}\left[-d \eta^{2}+\delta_{i j} d x^{i} d x^{j}\right]$, and the homogeneous scalar field $\phi_{0}(\eta)$. The evolution equations for this background are scalar field equations,

$$
\ddot{\phi}_{0}+2 \frac{\dot{a}}{a} \dot{\phi}_{0}+a^{2} \partial_{\phi} V[\phi]=0, \quad 3 \frac{\dot{a}^{2}}{a^{2}}=4 \pi G\left(\dot{\phi}_{0}^{2}+2 a^{2} V\left[\phi_{0}\right]\right) .
$$

The scale factor solution corresponding to the inflationary era of standard inflationary cosmology, written using a conformal time, is: $a(\eta)=-1 /\left[H_{I}^{2}(1-\epsilon) \eta\right]$ with $H_{I}^{2} \simeq(8 \pi / 3) G V, \epsilon$ is the slow-roll parameter which during the inflationary stage is considered to be very small $\epsilon \ll 1$, thus $H_{I} \simeq$ const, and with the scalar field $\phi_{0}$ in the slow roll regime, i.e. $\dot{\phi}_{0}=-\left(a^{3} / 3 \dot{a}\right) V^{\prime}$. According to the standard inflationary scenario, this era is followed by a reheating period in which the Universe is repopulated with ordinary matter fields, a regime that then evolves towards a standard hot big bang cosmology regime leading up to the present cosmological time. The functional form of $a(\eta)$ during these latter periods changes, but we will ignore those details because most of the change in the value of $a$ occurs during the inflationary regime. We will set $a=1$ at the "present cosmological time", and assume that inflationary regime ends at a value of $\eta=\eta_{R}$, negative and very small in absolute terms $\left(\eta_{R} \simeq-10^{-22} \mathrm{Mpc}\right)$.

Next, we consider the perturbations. We will ignore vectorial perturbations, and focus on the scalar and tensorial ones. First, we will focus on the scalar perturbations. Working in the so called longitudinal gauge, the perturbed metric is written as:

$$
d s^{2}=a(\eta)^{2}\left[-(1+2 \Psi) d \eta^{2}+(1-2 \Psi) \delta_{i j} d x^{i} d x^{j}\right]
$$

where $\Psi$ stands for the scalar perturbation usually known as the Newtonian potential.

The perturbation of the scalar field is related to a perturbation of the energy momentum tensor, and reflected into Einstein's equations which at lowest order lead to the following constraint equation for the Newtonian potential:

$$
\nabla^{2} \Psi=4 \pi G \dot{\phi}_{0} \delta \dot{\phi}=s \delta \dot{\phi}
$$

where we introduced the abbreviation $s \equiv 4 \pi G \dot{\phi}_{0}$.

Regarding the field $\delta \phi$, it is convenient to work with the rescaled field variable $y=a \delta \phi$ and its conjugate momentum $\pi=\dot{y}-\frac{\dot{a}}{a} y=a \delta \dot{\phi}$. We consider the quantum theory of this field setting the problem, for simplicity, in a finite box of side $L$, which can be taken to $\infty$ at the end of all calculations. Thus we write the expansions for field and conjugate momentum operators as

$$
\hat{y}(\eta, \vec{x})=\frac{1}{L^{3}} \sum_{\vec{k}} e^{i \vec{k} \cdot \vec{x}} \hat{y}_{\vec{k}}(\eta), \quad \hat{\pi}(\eta, \vec{x})=\frac{1}{L^{3}} \sum_{\vec{k}} e^{i \vec{k} \cdot \vec{x}} \hat{\pi}_{\vec{k}}(\eta),
$$


where the sum is over the wave vectors $\vec{k}$ satisfying $k_{i} L=2 \pi n_{i}$ for $i=1,2,3$ with $n_{i}$ integer and where $\hat{y}_{\vec{k}}(\eta) \equiv y_{k}(\eta) \hat{a}_{\vec{k}}+y_{k}^{*}(\eta) \hat{a}_{-\vec{k}}^{\dagger}$ and $\hat{\pi}_{\vec{k}}(\eta) \equiv g_{k}(\eta) \hat{a}_{\vec{k}}+g_{k}^{*}(\eta) \hat{a}_{-\vec{k}}^{\dagger}$ with the usual choice of modes:

$$
y_{k}(\eta)=\frac{1}{\sqrt{2 k}}\left(1-\frac{i}{\eta k}\right) \exp (-i k \eta), \quad g_{k}(\eta)=-i \sqrt{\frac{k}{2}} \exp (-i k \eta),
$$

which leads to what is known as the Bunch-Davies vacuum.

Note that according to the point of view we discussed in Section 2.1, and having at this point the quantum theory for the relevant matter fields, the effects of the quantum fields on the geometrical variables are codified in the semiclassical Einstein's equations. Thus equation (1) must be replaced by

$$
\nabla^{2} \Psi=s\langle\hat{\dot{\phi}}\rangle=(s / a)\langle\hat{\pi}\rangle .
$$

At this point, one can clearly observe that if the state of the quantum field is in the vacuum state, the metric perturbations vanish and thus the space-time is homogeneous and isotropic.

The proposal is based on the consideration of a self induced collapse, which we take to operate in close analogy with a "measurement" (but evidently, with no external measuring apparatus or observer involved). This leads us to want to work with Hermitian operators, which in ordinary quantum mechanics are the ones susceptible of direct measurement. Therefore, we must separate both $\hat{y}_{\vec{k}}(\eta)$ and $\hat{\pi}_{\vec{k}}(\eta)$ into their real and imaginary parts $\hat{y}_{\vec{k}}(\eta)=\hat{y}_{\vec{k}}{ }^{R}(\eta)+i \hat{y}_{\vec{k}}{ }^{I}(\eta)$ and $\hat{\pi}_{\vec{k}}(\eta)=\hat{\pi}_{\vec{k}}{ }^{R}(\eta)+i \hat{\pi}_{\vec{k}}^{I}(\eta)$, where

$$
\hat{y}_{\vec{k}}^{R, I}(\eta)=\frac{1}{\sqrt{2}}\left(y_{k}(\eta) \hat{a}_{\vec{k}}^{R, I}+y_{k}^{*}(\eta) \hat{a}_{\vec{k}}^{R, I \dagger}\right), \quad \hat{\pi}_{\vec{k}}^{R, I}(\eta)=\frac{1}{\sqrt{2}}\left(g_{k}(\eta) \hat{a}_{\vec{k}}^{R, I}+g_{k}^{*}(\eta) \hat{a}_{\vec{k}}^{R, I \dagger}\right),
$$

with $\hat{a}_{\vec{k}}^{R} \equiv \frac{1}{\sqrt{2}}\left(\hat{a}_{\vec{k}}+\hat{a}_{-\vec{k}}\right), \hat{a}_{\vec{k}}^{I} \equiv \frac{-i}{\sqrt{2}}\left(\hat{a}_{\vec{k}}-\hat{a}_{-\vec{k}}\right)$. Then the operators $\hat{y}_{\vec{k}}^{R, I}(\eta)$ and $\hat{\pi}_{\vec{k}}^{R, I}(\eta)$ are hermitian ${ }^{2}$.

So far, the treatment is similar to the standard one, except in that only the scalar field is being treated at the quantum level, while the metric fluctuation is treated classically. At this point, it is worthwhile to emphasize that the vacuum state defined by $\hat{a}_{\vec{k}}{ }^{R, I}|0\rangle=0$ is $100 \%$ translational and rotationally invariant, as can be seen from the fact that it is annihilated by the generators of translations and rotations (i.e. by the linear and the angular momentum operators, $\hat{\vec{P}}$ and $\hat{\vec{L}}$, respectively).

Next we need to specify in more detail the modeling of the collapse. Then, take into account that after the collapse has taken place, we must consider the continuing evolution of the expectation values of the field variables until the end of inflation, and eventually up to the last scattering hypersurface (in fact, if we want to actually compare our analysis with the detailed observations, we must evolve also through the reheating period and through the decoupling era up to today's Universe. This, however, is normally taken into account through the use of appropriate transfer functions, and we will assume that the same procedure can be implemented after the present analysis).

We will further assume that the collapse is somehow analogous to an imprecise measurement ${ }^{3}$ of the operators $\hat{y}_{\vec{k}}^{R, I}(\eta)$ and $\hat{\pi}_{\vec{k}}^{R, I}(\eta)$. Now, we will specify the rules according to which the

\footnotetext{
${ }^{2}$ Note however that he commutation relations of the real and imaginary creation and annihilation operators are however non-standard: $\left[\hat{a}_{\vec{k}}^{R}, \hat{a}_{\vec{k}^{\prime}}^{R, \dagger}\right]=\hbar L^{3}\left(\delta_{\vec{k}, \vec{k}^{\prime}}+\delta_{\vec{k},-\vec{k}^{\prime}}\right),\left[\hat{a}_{\vec{k}}^{I}, \hat{a}_{\vec{k}}^{I, \dagger}\right]=\hbar L^{3}\left(\delta_{\vec{k}, \vec{k}^{\prime}}-\delta_{\vec{k},-\vec{k}^{\prime}}\right)$, with all other commutators vanishing. This is known to indicate that the operators corresponding to $\vec{k}$ and $-\vec{k}$ are identical in the real case and identical up to a sign in the imaginary case.

${ }^{3}$ An imprecise measurement of an observable, is one in which one does not end with an exact eigenstate of that observable but rather with a state which is only peaked around the eigenvalue. Thus, we could consider measuring a certain particle's position and momentum, ending up with a state characterized by wave packet with both position and momentum defined to a limited extent, and which, of course, does not entail a conflict with Heisenberg's uncertainty bound.
} 
collapse happens. Again, at this point our criteria will be simplicity and naturalness. What we have to describe is the state $|\Theta\rangle$ after the collapse. It turns out that, for the goals at hand, all we need to specify is the expectation values immediately after the collapse as this determines the expectation value of the field and momentum operator for the mode $\vec{k}$ at all times after the collapse. That is using the result in the evolution equations for the expectation values (i.e. using Ehrenfest's theorem), one obtains $\left\langle\hat{y}_{\vec{k}}^{R, I}(\eta)\right\rangle$ and $\left\langle\hat{\pi}_{\vec{k}} R, I(\eta)\right\rangle$ for the state that resulted from the collapse, for all later times.

As we indicated, at this point we assume that after the collapse, the expectation values of the field and momentum operators in each mode will be related to the uncertainties of the precollapse state (recall that the expectation values in the vacuum state are zero). In the vacuum state, $\hat{y}_{\vec{k}}$ and $\hat{\pi}_{\vec{k}}$ individually are distributed according to Gaussian wave functions centered at 0 with spread $\left(\Delta \hat{y}_{\vec{k}}\right)_{0}^{2}$ and $\left(\Delta \hat{\pi}_{\vec{k}}\right)_{0}^{2}$, respectively.

We might consider various possibilities for the detailed form of this collapse. Thus, for their generic form, associated with the ideas above, we write:

$$
\begin{aligned}
& \left\langle\hat{y}_{\vec{k}}^{R, I}\left(\eta_{k}^{c}\right)\right\rangle_{\Theta}=\lambda_{y} x_{\vec{k}, y}^{R, I} \sqrt{\left(\Delta \hat{y}_{\vec{k}}^{R, I}\right)_{0}^{2}}=\lambda_{y} x_{\vec{k}, y}^{R, I}\left|y_{k}\left(\eta_{k}^{c}\right)\right| \sqrt{\hbar L^{3} / 2}, \\
& \left\langle\hat{\pi}_{\vec{k}}^{R, I}\left(\eta_{k}^{c}\right)\right\rangle_{\Theta}=\lambda_{\pi} x_{\vec{k}, \pi}^{R, I} \sqrt{\left(\Delta \hat{\pi}_{\vec{k}}^{R, I}\right)_{0}^{2}},=\lambda_{\pi} x_{\vec{k}, \pi}^{R, I}\left|g_{k}\left(\eta_{k}^{c}\right)\right| \sqrt{\hbar L^{3} / 2}
\end{aligned}
$$

where $\eta_{\vec{k}}^{c}$ represents the time of collapse for each mode. The random variables $x_{\vec{k}, y}^{R, I}, x_{\vec{k}, \pi}^{R, I}$ are selected randomly from a distribution which, in principle, might be non-Gaussian, i.e. different modes could present particular correlations (we will elaborate on this in the next section). At this point, we must emphasize that our Universe corresponds to a single realization of these random variables, and thus each of these quantities $x_{\vec{k}, y}^{R, I}, x_{\vec{k}, \pi}^{R, I}$ have a single specific value. The two parameters $\lambda_{y}$ and $\lambda_{\pi}$ are real numbers we use to characterize the different collapse schemes, e.g.: i) $\lambda_{y}=0, \lambda_{\pi}=1$, ii) $\lambda_{y}=\lambda_{\pi}=1$ (which we call the "symmetric scheme"). It is clear that one can devise many other models of collapse, a good fraction of them can be described within the scheme above, while others require a slightly modified treatment [5]. Still, there are surely many other possibilities which we have not even thought about and which might require drastically modified formalisms.

The explicit expressions for the $\left\langle\hat{y}_{\vec{k}}^{R, I}(\eta)\right\rangle_{\Theta}$ and $\left\langle\hat{\pi}_{\vec{k}}^{R, I}(\eta)\right\rangle_{\Theta}$ are then

$$
\begin{aligned}
\left\langle\hat{y}_{\vec{k}}^{R, I}(\eta)\right\rangle_{\Theta}= & {\left[\frac{\cos D_{k}}{k}\left(\frac{1}{k \eta}-\frac{1}{z_{k}}\right)+\frac{\sin D_{k}}{k}\left(\frac{1}{k \eta z_{k}}+1\right)\right]\left\langle\hat{\pi}_{\vec{k}}^{R, I}\left(\eta_{\vec{k}}^{c}\right)\right\rangle_{\Theta} } \\
& +\left(\cos D_{k}-\frac{\sin D_{k}}{k \eta}\right)\left\langle\hat{y}_{\vec{k}}^{R, I}\left(\eta_{\vec{k}}^{c}\right)\right\rangle_{\Theta}, \\
\left\langle\hat{\pi}_{\vec{k}}^{R, I}(\eta)\right\rangle_{\Theta}= & \left(\cos D_{k}+\frac{\sin D_{k}}{z_{k}}\right)\left\langle\hat{\pi}_{\vec{k}}^{R, I}\left(\eta_{\vec{k}}^{c}\right)\right\rangle_{\Theta}-k \sin D_{k}\left\langle\hat{y}_{\vec{k}}^{R, I}\left(\eta_{\vec{k}}^{c}\right)\right\rangle_{\Theta},
\end{aligned}
$$

where $D_{k}(\eta) \equiv k \eta-z_{k}$ and $z_{k} \equiv k \eta_{\vec{k}}^{c}$.

With this information at hand we can now compute the perturbations of the metric after the collapse of all the modes ${ }^{4}$.

\subsection{The observable quantities}

Now, we must put together our semi-classical description of the gravitational degrees of freedom and the quantum mechanics description of the inflaton field. We recall that this entails the semiclassical version of the perturbed Einstein's equation that, in our case, leads to equation (2).

\footnotetext{
${ }^{4}$ In fact, we need only be concerned with the relevant modes, those that affect the observational quantities in a relevant way. Modes that have wavelengths that are either too large or too small are irrelevant in this sense.
} 
The Fourier components at the conformal time $\eta$ are given by:

$$
\Psi_{\vec{k}}(\eta)=-\sqrt{\frac{\epsilon}{2}} \frac{H_{I} \hbar}{M_{P} k^{2}}\left\langle\hat{\pi}_{\vec{k}}(\eta)\right\rangle,
$$

where we used that during inflation $s \equiv 4 \pi G \dot{\phi_{0}}=\sqrt{\epsilon / 2} a H_{I} / M_{P}$, with $M_{P}$ the reduced Planck's mass $M_{P}^{2} \equiv \hbar^{2} /(8 \pi G)$. The expectation value depends on the state of the quantum field, therefore, as we have already noted, prior to the collapse, we have $\Psi_{\vec{k}}(\eta)=0$, and the space-time is still homogeneous and isotropic at the corresponding scale. However, after the collapse takes place, the state of the field is a different state with new expectation values which generically will not vanish, indicating that after this time the Universe becomes anisotropic and in-homogeneous at the corresponding scale. We now can reconstruct the space-time value of the Newtonian potential using $\Psi(\eta, \vec{x})=\frac{1}{L^{3}} \sum_{\vec{k}} e^{i \vec{k} \cdot \vec{x}} \Psi_{\vec{k}}(\eta)$, to extract the quantities of observational interest.

In order to make contact with the observations, we shall relate the expression (7) for the evolution of the Newtonian potential during the early phase of accelerated expansion, to the small anisotropies observed in the temperature of the cosmic microwave background radiation, $\delta T(\theta, \varphi) / T_{0}$ with $T_{0} \approx 2.725 K$ the temperature average. They are considered as the fingerprints of the small perturbations pervading the Universe at the time of decoupling, and undoubtedly any model for the origin of the seeds of cosmic structure should account for them.

The observational data is described in terms of the coefficients $\alpha_{l m}$ of the multipolar series expansion

$$
\frac{\delta T}{T_{0}}(\theta, \varphi)=\sum_{l m} \alpha_{l m} Y_{l m}(\theta, \varphi), \quad \alpha_{l m}=\int \frac{\delta T}{T_{0}}(\theta, \varphi) Y_{l m}^{*}(\theta, \varphi) d \Omega .
$$

Here $\theta$ and $\varphi$ are the coordinates on the celestial two-sphere, with $Y_{l m}(\theta, \varphi)$ the spherical harmonics. The different multipole numbers $l$ correspond to different angular scales; low $l$ to large scales and high $l$ to small scales. At large angular scales $(l \lesssim 20)$ the anisotropies in the CMB arise due to the Sachs-Wolfe effect. That effect relates the anisotropies in the temperature observed today on the celestial sphere to the inhomogeneities in the Newtonian potential on the last scattering surface: $\frac{\delta T}{T_{0}}(\theta, \varphi)=\frac{1}{3} \Psi\left(\eta_{D}, \vec{x}_{D}\right)$, where $\eta_{D}$ is the conformal time of decoupling which lies in the matter-dominated epoch, and $\vec{x}_{D}=R_{D}(\sin \theta \sin \varphi, \sin \theta \cos \varphi, \cos \theta)$, with $R_{D}$ the radius of the last scattering surface. Furthermore, using $\Psi(\eta, \vec{x})=\frac{1}{L^{3}} \sum_{\vec{k}} e^{i \vec{k} \cdot \vec{x}} \Psi_{\vec{k}}(\eta)$ and $e^{i \vec{k} \cdot \vec{x}_{D}}=4 \pi \sum_{l m} i j_{l}\left(k R_{D}\right) Y_{l m}(\theta, \varphi) Y_{l m}^{*}(\hat{k})$, the expression (8) for $\alpha_{l m}$ can be rewritten in the form

$$
\alpha_{l m}=\frac{4 \pi i^{l}}{3 L^{3}} \sum_{\vec{k}} j_{l}\left(k R_{D}\right) Y_{l m}^{*}(\hat{k}) \Delta(k) \Psi_{\vec{k}}\left(\eta_{R}\right),
$$

with $j_{l}\left(k R_{D}\right)$ the spherical Bessel function of order $l$. The transfer function $\Delta(k)$ represents the evolution of the Newtonian potential from the end of inflation $\eta_{R}$ to the last scattering surface at the time of decoupling $\eta_{D}$, i.e. $\Psi_{\vec{k}}\left(\eta_{D}\right)=\Delta(k) \Psi_{\vec{k}}\left(\eta_{R}\right)$. We will be ignoring this aspect from this point onward, despite the fact that this transfer functions are behind the famous acoustic peaks, the most noteworthy feature of the CMB spectrum, as they relate to aspects of plasma physics that are well understood and thus uninteresting from our point of view. This will mean that, from our point of view, the observational spectrum would have such features removed before comparing with our results (this is in the same spirit that one removes the uninteresting imprint of our galaxy on the observations of the CMB, or the dipole associated with our peculiar motion). This would be equivalent to assume that the observed spectrum fits well with the flat Harrison-Zel'dovich spectrum and setting $\Delta(k)=1$, and thus setting for our purposes here $\Psi_{\vec{k}}\left(\eta_{D}\right)=\Psi_{\vec{k}}\left(\eta_{R}\right)$. 
Substituting (6) in (7) and using (3), (4) gives

$$
\begin{aligned}
\Psi_{\vec{k}}\left(\eta_{R}\right)= & \frac{-(L \hbar)^{3 / 2} \sqrt{\epsilon} H_{I}}{2 \sqrt{2} M_{P} k^{3 / 2}}\left[\lambda_{\pi}\left(\cos D_{k}+\frac{D_{k}}{z_{k}}\right)\left(x_{\vec{k}, \pi}^{R}+i x_{\vec{k}, \pi}^{I}\right)\right. \\
& \left.-\lambda_{y} \sin D_{k}\left(1+\frac{1}{z_{k}^{2}}\right)^{1 / 2}\left(x_{\vec{k}, y}^{R}+i x_{\vec{k}, y}^{I}\right)\right] .
\end{aligned}
$$

where $z_{k} \equiv k \eta_{\vec{k}}^{c}$, we note that the quantity $D_{k}$ is being evaluated at $\eta_{R}$, i.e. $D_{k}\left(\eta_{R}\right)=k \eta_{R}-z_{k}$.

Finally using, (10) in (9) yields:

$$
\begin{aligned}
\alpha_{l m}= & -\frac{\pi i^{l} \hbar^{3 / 2} \sqrt{2 \epsilon} H_{I}}{3 L^{3 / 2} M_{P}} \sum_{\vec{k}} \frac{j_{l}\left(k R_{D}\right)}{k^{3 / 2}} Y_{l m}^{*}(\hat{k})\left[\lambda_{\pi}\left(\cos D_{k}+\frac{\sin D_{k}}{z_{k}}\right)\left(x_{\vec{k}, \pi}^{R}+i x_{\vec{k}, \pi}^{I}\right)\right. \\
& \left.-\lambda_{y} \sin D_{k}\left(1+\frac{1}{z_{k}^{2}}\right)^{1 / 2}\left(x_{\vec{k}, y}^{R}+i x_{\vec{k}, y}^{I}\right)\right] .
\end{aligned}
$$

We will further simplify the treatment by noting that the value of $-k \eta_{R}$ is exceedingly small for the modes of interest. Thus, this will be ignored in comparison to $z_{k}$, i.e. we will be assuming that we can make the approximation $D_{k} \rightarrow-z_{k}$.

It is worthwhile to mention that the relation of $\alpha_{l m}$ with the Newtonian potential, as obtained in (11) within the collapse framework has no analogue in the usual treatments of the subject. It provides us with a clear identification of the aspects of the analysis where the "randomness" is located. In this case, it resides in the randomly selected values $x_{\vec{k}, y}^{\mathrm{R}, \mathrm{I}}, x_{\vec{k}, \pi}^{\mathrm{R}, \mathrm{I}}$ that appear in the expressions of the collapses associated with each of the modes. Here, we also find a clarification of how is it that, in spite of the intrinsic randomness, we can make any prediction at all. The individual complex quantities $\alpha_{l m}$ correspond to large sums of complex contributions, each one having a certain randomness, but leading in combination to a characteristic value, in just the same way as a random walk that is made up of multiple steps. In other words, the justification for the use of statistics in our approach is: The quantity $\alpha_{l m}$ is the sum of contributions from the collection of modes, each contribution being a random number leading to what is in effect a sort of "two-dimensional random walk", which total displacement corresponds to the observational quantity. Nothing like this can be found in the most popular accounts, in which the issues we have been focussing on are hidden in a maze of often unspecified assumptions and unjustified identifications [44].

Finally, let us discuss the implications of our proposal regarding the tensorial perturbations. The analysis we present is for the sake of completeness, as we have already presented it in previous works [24, 37], and is not the main subject of this article.

The view we take, regarding the relation between the metric (classical) description of gravity and the quantum matter degrees of freedom, has a direct implication on the estimates for the production of gravitational waves (from inflation). The idea is that the mechanism that generates the actual the fluctuations is tied to on the quantum uncertainties for the scalar field. Due to some unknown quantum gravitational effect, the state of each mode of the scalar field collapses. After the collapse, the density inhomogeneities and anisotropies feed into the gravitational degrees of freedom resulting in perturbations (scalar, vector and tensor) of the metric. Nevertheless, the metric itself does not induce a quantum gravitational collapse. Therefore, as the scalar field does not act as a source for the gravitational tensor modes - at least not at the order considered here - the tensor modes cannot be excited. Thus, the collapse scheme naturally predicts ${ }^{5}$ a zero - or at least a strongly suppressed - amplitude of gravitational waves into the CMB.

\footnotetext{
${ }^{5}$ However, it is worthwhile pointing out that such a conclusion is directly tied to our underlying approach that favors the semi-classical Einstein's equations augmented with a collapse proposal as a way to deal with the gravity
} 


\section{Modified statistical features in the primordial spectrum}

In this section, we will show how the statistical aspects of the collapse model might induce non-trivial features in the primordial spectrum. This is a novel aspect and contrasts with what has been done in the previous approaches, where the different modes that contribute to the primordial spectrum are considered to be uncorrelated.

Let us start by noting that according to (11) all the modes contribute to $\alpha_{l m}$ with a complex number. If we had the outcomes characterizing each of the individual collapses we could, of course, predict the value of these quantities. However, we have at this point no other access to such information than the observational quantities $\alpha_{l m}$ themselves.

We hope to be able to say something about these, but doing so requires the consideration of further hypothesis regarding the statistical aspects of the physics behind the collapse as well as the conditions previous to them.

As is generally the case with random walks, one can not hope to estimate the direction of the final displacement. However, one might say something about its estimated magnitude. It is for that reason we will be focussing on estimating the most likely value of the magnitude $\left|\alpha_{l m}\right|^{2}$. We can make some progress, for instance, by making some assumption allowing us to regard the specific outcomes characterizing our Universe as a typical member of some hypothetical ensemble of Universes.

For example, we are interested in estimating the most likely value of the magnitude of $\left|\alpha_{l m}\right|^{2}$ above, and in such hypothetical ensemble we might hope that it comes very close to our single sample. It is worthwhile emphasizing that, for each $l$ and $m$, we have one single complex number characterizing the actual observations (and thus the real Universe we inhabit). For a given $l$ for instance, we should avoid confusing ensemble averages with averages of such quantities over the $2 l+1$ values of $m$. The other universes in the ensemble are just constructs of our imagination and there is nothing in our theories that would indicate that they are real.

We can simplify things even further by taking the ensemble average $\overline{\left|\alpha_{l m}\right|^{2}}$ (recall that the bar represents an ensemble average) and identifying it with the most likely value of the quantity, and needless is to say that these two notions are different in many types of ensembles. However, let us, for the moment, ignore this issue and assume the identity of those two values. We thus have

$$
\begin{aligned}
& \left|\alpha_{l m}\right|_{\mathrm{ML}}^{2}=\overline{\left|\alpha_{l m}\right|^{2}}=\frac{2 \pi^{2} \hbar^{3} \epsilon H_{I}}{9 L^{3} M_{P}^{2}} \sum_{\vec{k}, \overrightarrow{k^{\prime}}} \frac{j_{l}\left(k R_{D}\right)}{k^{3 / 2}} \frac{j_{l}\left(k^{\prime} R_{D}\right)}{k^{\prime 3 / 2}} Y_{l m}^{*}(\hat{k}) Y_{l m}\left(\hat{k^{\prime}}\right) \\
& \times\left[\lambda_{\pi} M\left(z_{k}\right) M\left(z_{k^{\prime}}\right)\left(\overline{x_{\vec{k}, \pi}^{R} x_{\vec{k}^{\prime}, \pi}^{R}}+\overline{x_{\vec{k}, \pi}^{I} x_{\vec{k}^{\prime}, \pi}^{I}}\right)+\lambda_{y}^{2} N\left(z_{k}\right) N\left(z_{k^{\prime}}\right)\left(\overline{x_{\vec{k}, y}^{R} x_{\vec{k}^{\prime}, y}^{R}}+\overline{x_{\vec{k}, y}^{I} x_{\vec{k}^{\prime}, y}^{I}}\right)\right],
\end{aligned}
$$

with

$$
M\left(z_{k}\right) \equiv \cos z_{k}-\frac{\sin z_{k}}{z_{k}}, \quad N\left(z_{k}\right) \equiv \sin z_{k}\left(1+\frac{1}{z_{k}^{2}}\right)^{1 / 2}
$$

and we have used the assumption that the four groups of random variables $x_{\vec{k}, y}^{R}, x_{\vec{k}, y}^{I}, x_{\vec{k}, \pi}^{R}, x_{\vec{k}, \pi}^{I}$ are completely uncorrelated (leading thus to the cancelation of the cross terms in (12)).

As mentioned in Section 2.1, our refined approach suggests that, within each set of random variables, there would be a correlation between the modes $\vec{k}$ and $2 \vec{k}$ (and also of course between $\vec{k}$

quantum interface faced in the current problem. It is of course conceivable, although seems harder to understand in a wider context (see the discussion in Section 8 of [43]), that a collapse might be incorporated into a setting where both the gravitation and scalar field perturbations are simultaneously treated at the quantum level. If the latter happened to be the correct approach, something that would be possible to ascertain when we have a fully satisfactory theory of quantum gravity, our conclusion about the tensor modes would be modified. 
and $\vec{k} / 2$ ). This correlation would induce a "small" departure from the normal probability distribution function of the random variables, where the "smallness" is characterized by the introduction of a parameter $\varepsilon$ (do not confuse $\varepsilon$ with the slow-roll parameter $\epsilon$ ). That is, we would assume that the ensemble average of the product of two random variables is given by

$$
\begin{aligned}
& \overline{x_{\vec{k}, i}^{R} x_{\vec{k}^{\prime}, i}^{R}}=\delta_{\vec{k}, \vec{k}^{\prime}}+\delta_{\vec{k},-\vec{k}^{\prime}}+\varepsilon\left(\delta_{\vec{k}, 2 \vec{k}^{\prime}}+\delta_{\vec{k},-2 \vec{k}^{\prime}}+\delta_{2 \vec{k}, \vec{k}^{\prime}}+\delta_{-2 \vec{k}, \vec{k}^{\prime}}\right), \\
& \overline{x_{\vec{k}, i}^{I} x_{\vec{k}^{\prime}, i}^{I}}=\delta_{\vec{k}, \vec{k}^{\prime}}-\delta_{\vec{k},-\vec{k}^{\prime}}+\varepsilon\left(\delta_{\vec{k}, 2 \vec{k}^{\prime}}-\delta_{\vec{k},-2 \vec{k}^{\prime}}+\delta_{2 \vec{k}, \vec{k}^{\prime}}-\delta_{-2 \vec{k}, \vec{k}^{\prime}}\right),
\end{aligned}
$$

with $i=y, \pi$. Note that this suggests also many more complex and higher order correlations between various sets of variables, but we will neglect those higher order effects here, in part because we do not have a specific self-content mold for those at this point. We recall that the random variables corresponding to $\vec{k}$ and $-\vec{k}$ are not independent. Using (13) and (14) in (12) we obtain

$$
\begin{aligned}
\left|\alpha_{l m}\right|_{\mathrm{ML}}^{2}= & \frac{4 \pi^{2} \hbar^{3} \epsilon H_{I}}{9 L^{3} M_{P}^{2}} \sum_{\vec{k}, \vec{k}^{\prime}} \frac{j_{l}\left(k R_{D}\right)}{k^{3 / 2}} \frac{j_{l}\left(k^{\prime} R_{D}\right)}{k^{\prime 3 / 2}} Y_{l m}^{*}(\hat{k}) Y_{l m}\left(\hat{k}^{\prime}\right) \\
& \times\left[\lambda_{\pi}^{2} M\left(z_{k}\right) M\left(z_{k^{\prime}}\right)+\lambda_{y}^{2} N\left(z_{k}\right) N\left(z_{k^{\prime}}\right)\right]\left[\delta_{\vec{k}, \vec{k}^{\prime}}+\varepsilon\left(\delta_{\vec{k}, 2 \vec{k}^{\prime}}+\delta_{2 \vec{k}, \vec{k}^{\prime}}\right)\right] .
\end{aligned}
$$

As we have found in previous works, the data indicates that, to a good degree of accuracy [5], the assumption that $z_{k}$ is independent of $k$, i.e. the time of collapse goes as $\eta_{\vec{k}}^{c} \propto k^{-1}$, is a good approximation. Therefore, assuming $z_{k}=z=$ const, (15) takes the form

$$
\begin{aligned}
\left|\alpha_{l m}\right|_{\mathrm{ML}}^{2}= & \frac{4 \pi^{2} \hbar^{3} \epsilon H_{I} C(z)}{9 L^{3} M_{P}^{2}}\left\{\sum _ { \vec { k } } \frac { | Y _ { l m } ( \hat { k } ) | ^ { 2 } } { k ^ { 3 } } \left[j_{l}^{2}\left(k R_{D}\right)+\varepsilon\left(2^{3 / 2} j_{l}\left(k R_{D}\right) j_{l}\left(k R_{D} / 2\right)\right.\right.\right. \\
& \left.\left.\left.+2^{-3 / 2} j_{l}\left(k R_{D}\right) j_{l}\left(2 k R_{D}\right)\right)\right]\right\},
\end{aligned}
$$

where $C(z) \equiv \lambda_{\pi}^{2} M^{2}(z)+\lambda_{y}^{2} N^{2}(z)$. Now we write the sum as an integral by noting that the allowed values of the components of $\vec{k}$ are separated by $\Delta k_{i}=2 \pi / L$, that is

$$
\begin{aligned}
\left|\alpha_{l m}\right|_{\mathrm{ML}}^{2}= & \frac{\hbar^{3} \epsilon H_{I} C(z)}{18 \pi M_{P}^{2}}\left[\int_{0}^{\infty} \frac{d k}{k} j_{l}^{2}\left(k R_{D}\right)\right. \\
& \left.+\varepsilon \int_{0}^{\infty} \frac{d k}{k}\left(2^{3 / 2} j_{l}\left(k R_{D}\right) j_{l}\left(k R_{D} / 2\right)+2^{-3 / 2} j_{l}\left(k R_{D}\right) j_{l}\left(2 k R_{D}\right)\right)\right],
\end{aligned}
$$

where in the previous expression we performed the integral over the angular part of $\vec{k}$.

Rewriting the remaining integrals, we find

$$
\left|\alpha_{l m}\right|_{\mathrm{ML}}^{2}=\frac{\hbar^{3} \epsilon H_{I} C(z)}{18 \pi M_{P}^{2}}\left[\int_{0}^{\infty} \frac{d x}{x} j_{l}^{2}(x)+\frac{9}{2^{3 / 2}} \varepsilon \int_{0}^{\infty} \frac{d x}{x} j_{l}(x) j_{l}(2 x)\right],
$$

where we use a change of variable $x \equiv k R_{D}$. Evaluating the integrals of the products of spherical Bessel's functions gives the final result

$$
\left|\alpha_{l m}\right|_{\mathrm{ML}}^{2}=\frac{\hbar^{3} \epsilon H_{I} C(z)}{18 \pi M_{P}^{2}}\left[\frac{1}{2 l(l+1)}+\varepsilon \frac{9 \sqrt{\pi} \Gamma(l)}{2^{7 / 2+l} \Gamma(l+3 / 2)}{ }_{2} F_{1}(l,-1 / 2 ; l+3 / 2,1 / 4)\right],
$$

where $\Gamma(l)$ is the Gamma function, and ${ }_{2} F_{1}(\alpha, \beta ; \gamma, z)$ is the hypergeometric function ${ }^{6}$.

\footnotetext{
${ }^{6}$ The hypergeometric function is defined as ${ }_{2} F_{1}(\alpha, \beta ; \gamma, z) \equiv \frac{\Gamma(\gamma)}{\Gamma(\beta) \Gamma(\gamma-\beta)} \int_{0}^{1} \frac{t^{\beta-1}(1-t)^{\gamma-\beta-1}}{(1-t z)^{\alpha}} d t$.
} 


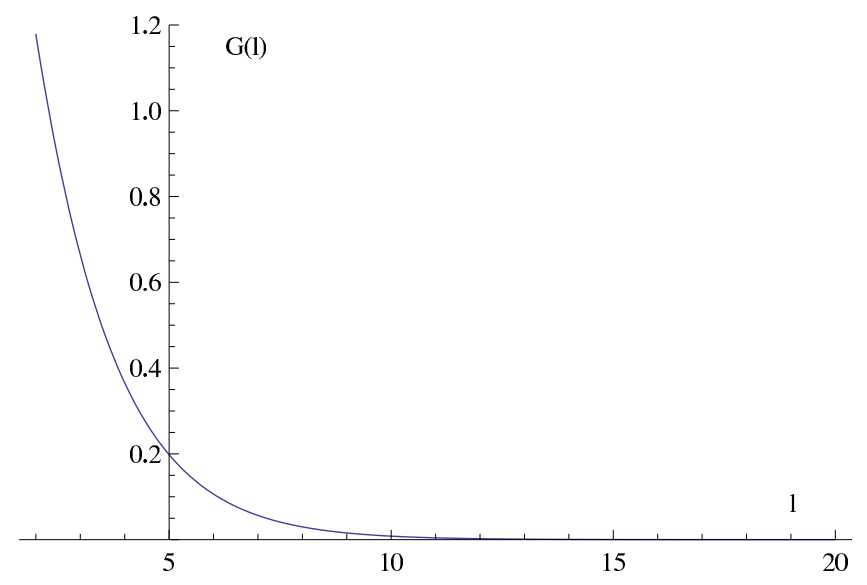

Figure 1. $G(l)$ for $l \in[2,20]$. The deviation from the flat spectrum will only affect the low multipoles.

Now, as (16) does not depend on $m$ it is clear that the expectation of the quantity of observational interest, i.e. the angular spectrum $l(l+1) C_{l} \equiv l(l+1)(2 l+1)^{-1} \sum_{m}\left|\alpha_{l m}\right|^{2}$, is just $\left|\alpha_{l m}\right|^{2}$ and thus this quantity must be compared with:

$$
l(l+1) C_{l}=\mathcal{A}[1+\varepsilon G(l)]
$$

where we have defined

$$
G(l) \equiv \frac{l(l+1) 9 \sqrt{\pi} \Gamma(l)}{2^{5 / 2+l} \Gamma(l+3 / 2)}{ }_{2} F_{1}(l,-1 / 2 ; l+3 / 2,1 / 4),
$$

and $\mathcal{A}$ is a normalization constant that is of no interest to us here. Clearly, if $\varepsilon=0$, which corresponds to the case that the probability distribution functions for the variables $x_{\vec{k}, i}^{R}, x_{\overrightarrow{k^{\prime}}, i}^{R}$ and $x_{\vec{k}, i}^{I}, x_{\vec{k}^{\prime}, i}^{I}$ are uncorrelated, random, and Gaussian (see (13) and (14)), then we recover the standard flat spectrum, i.e. $l(l+1) C_{l}$ is independent of $l$. The departure of the flat spectrum has a very specific signature that, in principle, can be searched for observationally. There are of course many other possibilities of unexpected correlations and each one will have a particular signature.

The behavior of $G(l)$ for $2 \leq l \leq 20$ is shown in Fig. 1 . As we can see, the effect is stronger for low $l$ and it decreases in a nearly exponential fashion for large $l$ (we have made a direct confirmation of this fact). The next step, of a more rigorous analysis, would be to consider the acoustic oscillations, due to plasma physics, which play an important role after the end of the inflationary regime. Then, we could use the recent observational data available (e.g. WMAP7), and thus put some constraints on the parameter $\varepsilon$.

Recall, however, that the result (17) we obtained depends on the behavior of another parameter we have introduced, namely the time of collapse $\eta_{\vec{k}}^{c}$. Let us recall, that in order to recover the detailed form of the spectrum, we have had to make the additional assumption that $\eta_{\vec{k}}^{c} \propto 1 / k$ (i.e. $z_{k}$ is independent of $k$ ). At this point we only have a very heuristic proposal that seems to account for this assumption (see [37], in terms of an argument that attempts to connect that pattern with certain ideas of Penrose's and Diosi's linking the collapse of the wave function with aspects of quantum gravity). In fact, the assumption that $\eta_{\vec{k}}^{c} \propto 1 / k$ seems necessary to recover something close to the observed spectral shape, as has already been noted in previous works $[5,37]$. The point is that, in order to constrain the values of $\varepsilon$ and $z_{k}$, we should perform an analysis such as the one presented in [22]. That work, consisted of a detailed statistical analysis testing some specific "collapse schemes", and establishing constraints on the free parameters of the modes considered. The analysis was carried out comparing the details 
predictions obtained by a modified $\mathrm{CMB}$ fast code incorporating out models, and recent data from the CMB, including the 7-yr data release of the WMAP [23] and the matter power spectrum measured employing the Luminous Red Galaxies by the Sloan Digital Sky Survey [38]. A similar analysis for the results of the present paper lies outside the scope of this work. However, our main result of this work, i.e. the pattern noted in (17), indicates that the appearance of the correlations between the modes $\vec{k}$ and $2 \vec{k}$; affecting the statistics of the random variables

$x_{\vec{k}, i}^{R}, x_{\vec{k}^{\prime}, i}^{R}$ and $x_{\vec{k}, i}^{I}, x_{\vec{k}^{\prime}, i}^{I}$, and thus our estimates for the observational quantities. It seems that looking for such specific pattern of deviation in the form of CMB spectrum, would be a straight forward (but highly resource consuming) computational endeavor.

Finally, let us end this section by noting a heuristic relation between our result (17) and the so called "parametric resonance" effect in quantum optics. The set up involves a parametric nonlinear crystal excited by an intense laser beam at angular frequency $\omega_{p}=2 \omega$. The interesting fact is that a weak signal beam at angular frequency $\omega$ is spontaneously generated. In a particular setting, the nonlinear crystal can mix a second input signal $\omega_{s}=\omega$ with that resulting from the pump, and this produces an idler beam at angular frequency $\omega$. The nonlinear process either amplifies or de-amplifies the excitation of the signal modes depending on the phases. Thus, the output field is the result of delicate quantum interference resulting from a highly correlated state. In short, the nonlinear nature of the crystal's response leads to photons of one frequency being converted into photons with a different one, leading to the appearance in the spectrum of the beam of important connections between the components with a given frequency and its first harmonics. The connection with our result (17) would lead us to view the gravitational response to the collapse of matter fields, as described by the SSC scheme, playing the role of the "non-linear crystal". That is, the collapse seems to correlate the mode $\vec{k}$ the modes $n \vec{k}$, ( $n$ an integer), in the same sense as the crystal correlates the angular frequency of the signal and the pump. The term proportional to $\varepsilon$ in (17) would correspond to the modification on the amplitude in the previous example.

\section{Discussion}

The work presented here is a continuation of a long standing program aimed at addressing and proposing a possible cure for a set of shortcomings that afflict the standard accounts for the emergence of the seeds of cosmic structure during inflation. The program has revealed that, although the motivating problems can be characterized as conceptual difficulties, it is intimately connected with what is usually called the measurement problem in quantum mechanics. This problem, which as we have argued, becomes essentially insurmountable within the standard quantum theory in the cosmological context (see [44]). Nevertheless, one is led surprisingly to expect observational consequences associated with some details of the physical unknown mechanisms that lie beyond quantum theory and our current understanding. That is, by parameterizing some aspects of this enigmatic process, we might actually use observations as a guides in learning something about them. In previous works, we have seen, for instance, that the details of the "collapse schemes" can modified the primordial fluctuation spectral shape in very particular ways [37]. We have argued that we can use the observations to place limits on the times of collapse of the various modes [5].

We have discussed that the semiclassical setting should be the appropriate framework to deal with inflationary perturbations. This is simply because the full quantum theory is likely to be one where the space-time notions are not present at all (due in part by the problem of time that afflicts canonical approaches to quantum gravity such as LQG). As has been argued elsewhere in that setting, there would not be tensor modes exited at first order in perturbation theory and thus no primordial gravity waves of a magnitude that could be observed in the forceable future. 
Moreover, in the approach we have been using in this and previous works, it becomes quite transparent on how the statistical aspects are related to each one of the observational quantities. That is, the observable $\alpha_{l m}$ is related to the random variables $x_{\vec{k}}$ 's through a complex "random walk", while the value of $\left|\alpha_{l m}\right|^{2}$ corresponds to the squared "length" of the random walk. This random walk is associated to a particular realization of a physical quantum process (i.e. associated with one of the multiple possibilities characterized by the collapse of the inflaton's wave-function). However, as we have access to just one such realization - the set of random walks corresponding to our own Universe - the most natural course of action (but certainly not the only one) is to take the ensemble average value of the length of the possible random walks, which corresponds to $\overline{\left|\alpha_{l m}\right|^{2}}$, to be identified with the most likely value, i.e. with $\left|\alpha_{l m}\right|_{\mathrm{ML}}^{2}$; and this in turn, is to be compared with the $\left|\alpha_{l m}\right|^{2}$ of our observable Universe. Of course, our Universe is to be identified with one realization of the random variables, and as such, the resulting picture has an interpretational transparency that seems hard to find in the standard accounts.

The clarification mentioned above has allowed us to take a fresh approach in addressing the possibility of novel statistical features and the manner in which those could be searched for, as discussed in [25]. In the present work we have discussed a novel possibility for nonstandard statistics uncovered by our approach. This novel aspect is tied to the possibility that non trivial statistical features would arise as a result of the of the collapse process itself. Namely that the collapse of one of the modes (say the mode $\vec{k}$ ) might influence the tendency in which the state of a related mode (say the mode $2 \vec{k}$ ) to collapse in a certain manner. We can heuristically view this possibility as reflecting a modification of the statistical aspects of the state of one mode as the result of the collapse of another.

We noted that the deviation from the standard prediction would be particularly relevant for the first multipoles: It is thus interesting to speculate whether this effect could be linked to the issues raised in $[4,39]$. In those works, it has been argued that there are specific large-scale anomalies in the temperature map of anisotropies of the CMB for the low modes. According to [39], the observations disagree markedly with the predictions of the standard theory. By that as it might, it seems, that our result (17), which was motivated by purely conceptual issues, shares some of the essential features of those observations, i.e. a departure of the standard flat - spectrum for the lowest $l$. Evidently, at this stage of the analysis we cannot present more quantitative results, however, we believe that the previous discussion serves to show the potential behind our proposal.

In any event, we have seen that such kind of modifications have particular signatures which could, in principle, be searched for in the data, leading as we have said, either to interesting discoveries, or at least to the setting of bounds on the type of effect we have been considering.

\section{Acknowledgements}

The work of GL and DS was supported in part by the CONACYT grant No 101712. GL acknowledges financial support by CONACYT postdoctoral grant. DS was also supported by the PAPIIT-UNAM grant IN107412. We thank the referees for useful suggestions.

\section{References}

[1] Albrecht A., Steinhardt P.J., Cosmology for grand unified theories with radiatively induced symmetry breaking, Phys. Rev. Lett. 48 (1982), 1220-1223.

[2] Bartolo N., Komatsu E., Matarrese S., Riotto A., Non-Gaussianity from inflation: theory and observations, Phys. Rep. 402 (2004), 103-266, astro-ph/0406398.

[3] Bassi A., Ghirardi G.C., Dynamical reduction models, Phys. Rep. 379 (2003), 257-426, quant-ph/0302164.

[4] Copi C.J., Huterer D., Schwarz D.J., Starkman G.D., Large angle anomalies in the CMB, Adv. Astron. 2010 (2010), 847541, 17 pages, arXiv:1004.5602. 
[5] De Unánue A., Sudarsky D., Phenomenological analysis of quantum collapse as source of the seeds of cosmic structure, Phys. Rev. D $\mathbf{7 8}$ (2008), 043510, 15 pages, arXiv:0801.4702.

[6] Diez-Tejedor A., León G., Sudarsky D., The collapse of the wave function in the joint metric-matter quantization for inflation, arXiv:1106.1176.

[7] Diez-Tejedor A., Sudarsky D., Towards a formal description of the collapse approach to the inflationary origin of the seeds of cosmic structure, arXiv:1108.4928.

[8] Diósi L., A universal master equation for the gravitational violation of quantum mechanics, Phys. Lett. A 120 (1987), 377-381.

[9] Diósi L., Models for universal reduction of macroscopic quantum fluctuations, Phys. Rev. A 40 (1989), $1165-1174$.

[10] Gambini R., Porto R.A., Pullin J., Fundamental decoherence from relational time in discrete quantum gravity: Galilean covariance, Phys. Rev. D 70 (2004), 124001, 8 pages, gr-qc/0408050.

[11] Gambini R., Porto R.A., Pullin J., Realistic clocks, universal decoherence, and the black hole information paradox, Phys. Rev. Lett. 93 (2004), 240401, 3 pages, hep-th/0406260.

[12] Ghirardi G.C., Rimini A., Weber T., Unified dynamics for microscopic and macroscopic systems, Phys. Rev. D 34 (1986), 470-491.

[13] Guth A.H., Inflationary universe: a possible solution to the horizon and flatness problems, Phys. Rev. D 23 (1981), 347-356.

[14] Guth A.H., Pi S.Y., Fluctuations in the new inflationary universe, Phys. Rev. Lett. 49 (1982), $1110-1113$.

[15] Guth A.H., Pi S.Y., Quantum mechanics of the scalar field in the new inflationary universe, Phys. Rev. D 32 (1985), 1899-1920.

[16] Halliwell J.J., Hawking S.W., Origin of structure in the universe, Phys. Rev. D 31 (1985), 1777-1791.

[17] Hawking S.W., The development of irregularities in a single bubble inflationary universe, Phys. Lett. B 115 (1982), 295-297.

[18] Hawking S.W., Moss I.G., Fluctuations in the inflationary universe, Nuclear Phys. B 224 (1983), 180-192.

[19] Isham C.J., Canonical quantum gravity and the problem of time, in Integrable Systems, Quantum Groups, and Quantum Field Theories (Salamanca, 1992), NATO Adv. Sci. Inst. Ser. C Math. Phys. Sci., Vol. 409, Kluwer Acad. Publ., Dordrecht, 1993, 157-287, gr-qc/9210011.

[20] Komatsu E., Hunting for primordial non-Gaussianity in the cosmic microwave background, Classical Quantum Gravity 27 (2010), 124010, 26 pages, arXiv:1003.6097.

[21] Komatsu E., The pursuit of non-gaussian fluctuations in the cosmic microwave background, Ph.D. thesis, Tohoku University, 2001, astro-ph/0206039.

[22] Landau S.J., Scoccola C.G., Sudarsky D., Cosmological constraints on non-standard inflationary quantum collapse models, arXiv:1112.1830.

[23] Larson D., Dunkley J., Hinshaw G. et al., Seven-year Wilkinson microwave anisotropy probe (WMAP) observations: power spectra and WMAP-derived parameters, Astrophys. J. Suppl. 192 (2011), 16, 19 pages, arXiv:1001.4635.

[24] León G., De Unánue A., Sudarsky D., Multiple quantum collapse of the inflaton field and its implications on the birth of cosmic structure, Classical Quantum Gravity 28 (2011), 155010, 22 pages, arXiv:1012.2419.

[25] León G., Landau S.J., Sudarsky D., Quantum origin of the primordial fluctuation spectrum and its statistics, arXiv:1107.3054.

[26] León G., Sudarsky D., The slow-roll condition and the amplitude of the primordial spectrum of cosmic fluctuations: contrasts and similarities of the standard account and the 'collapse scheme', Classical Quantum Gravity 27 (2010), 225017, 23 pages, arXiv:1003.5950.

[27] Liguori M., Sefusatti E., Fergusson J.R., Shellard E.P.S., Primordial non-Gaussianity and bispectrum measurements in the cosmic microwave background and large-scale structure, Adv. Astron. 2010 (2010), 980523, 64 pages, arXiv:1001.4707.

[28] Linde A.D., Coleman-Weinberg theory and the new inflationary universe scenario, Phys. Lett. B 114 (1982), 431-435.

[29] Lyth D.H., Liddle A.R., The primordial density perturbation: cosmology, inflation and the origin of structure, Cambridge University Press, Cambridge, 2009.

[30] Mukhanov V., Physical foundations of cosmology, Cambridge University Press, New York, 2005. 
[31] Mukhanov V.F., Chibisov G.V., Quantum fluctuation and nonsingular universe, JETP Lett. 33 (1981), 532-535.

[32] Mukhanov V.F., Feldman H.A., Brandenberger R.H., Theory of cosmological perturbations, Phys. Rep. 215 (1992), 203-333.

[33] Padmanabhan T., Structure formation in the universe, Cambridge University Press, New York, 1993.

[34] Pearle P.M., Dynamical wave function collapse: could it have cosmological consequences?, arXiv:0710.0567.

[35] Penrose R., On gravity's role in quantum state reduction, Gen. Relativity Gravitation 28 (1996), 581-600.

[36] Penrose R., The emperor's new mind. Concerning computers, minds, and the laws of physics, The Clarendon Press, Oxford University Press, New York, 1989.

[37] Perez A., Sahlmann H., Sudarsky D., On the quantum origin of the seeds of cosmic structure, Classical Quantum Gravity 23 (2006), 2317-2354, gr-qc/0508100.

[38] Reid B.A., Percival W.J., Eisenstein D.J. et al., Cosmological constraints from the clustering of the sloan digital sky survey DR7 luminous red galaxies, Mon. Not. Roy. Astron. Soc. 404 (2010), 60-85, arXiv:0907.1659.

[39] Starkman G.D., Copi C.J., Huterer D., Schwarz D., The oddly quiet universe: how the CMB challenges cosmology's standard model, arXiv:1201.2459.

[40] Starobinsky A.A., A new type of isotropic cosmological models without singularity, Phys. Lett. B 91 (1980), 99-102.

[41] Starobinsky A.A., Dynamics of phase transition in the new inflationary universe scenario and generation of perturbations, Phys. Lett. B 117 (1982), 175-178.

[42] Sudarsky D., A signature of quantum gravity at the source of the seeds of cosmic structure?, J. Phys. Conf. Ser. 67 (2007), 012054, 6 pages, gr-qc/0701071.

[43] Sudarsky D., Can we learn something about the quantum/gravity interface from the primordial fluctuation spectrum?, Internat. J. Modern Phys. D 20 (2011), 821-838.

[44] Sudarsky D., Shortcomings in the understanding of why cosmological perturbations look classical, Internat. J. Modern Phys. D 20 (2011), 509-552, arXiv:0906.0315.

[45] Sudarsky D., The seeds of cosmic structure as a door to new physics, J. Phys. Conf. Ser. 68 (2007), 012029, 12 pages, gr-qc/0612005.

[46] Wald R.M., Quantum field theory in curved spacetime and black hole thermodynamics, Chicago Lectures in Physics, University of Chicago Press, Chicago, IL, 1994.

[47] Weinberg S., Cosmology, Oxford University Press, Oxford, 2008.

[48] Yadav A.P.S., Wandelt B.D., Primordial non-Gaussianity in the cosmic microwave background, Adv. Astron. 2010 (2010), 565248, 27 pages, arXiv:1006.0275. 